\title{
Changing the Concept: From the Traditional Glucose-centric to the New Cardiorenal-metabolic Approach for the Treatment of Type 2 Diabetes
}

Dimitrios G Chatzis, ${ }^{1}$ Konstantinos Kolokathis, ${ }^{2}$ Kalliopi Magounaki, ${ }^{1}$ Stefanos Chatzidakis, ${ }^{1}$ Konstantinos Avramidis, ${ }^{2}$ Marianna Leopoulou, ${ }^{3}$ Theodoros P Angelopoulos ${ }^{4}$ and John Doupis ${ }^{2,4}$

1. European University of Cyprus Medical School, Nicosia, Cyprus; 2. Department of Internal Medicine and Diabetes, Salamis Naval and Veterans Hospital, Salamis Naval Base, Salamis, Attiki, Greece; 3. Department of Cardiology, "Elpis" General Hospital, Athens, Greece; 4. Iatriko Paleou Falirou Medical Center, Athens, Greece

DOI: https://doi.org/10.17925/EE.2021.17.2.92

$\mathrm{T}$ ype 2 diabetes mellitus (T2DM) is a chronic disease with a constantly increasing prevalence worldwide. It is well established that T2DM affects both the macro- and microvasculature, and its presence is associated with a high risk of acute and chronic cardiovascular events. Traditionally, the management of T2DM has been mainly focused on the optimization of blood glucose levels with the use of antidiabetic medications. During recent years, however, an impressive accumulation of evidence has arisen from studies designed to explore the plausible effects of new antidiabetic drugs on cardiovascular outcomes in patients with diabetes. This review article aims to emphasize the findings of these studies and to highlight the substantial role of the newer classes of antidiabetic drugs in treating T2DM in a holistic, cardiorenal-metabolic approach, thus shifting the paradigm from the traditional, simplistic, glucose-lowering approach.

\section{Keywords}

Type 2 diabetes mellitus (T2DM), treatment, glucose-lowering, cardiorenal-metabolic

Disclosures: Dimitrios G Chatzis, Konstantinos Kolokathis, Kalliopi Magounaki, Stefanos Chatzidakis, Konstantinos Avramidis, Marianna Leopoulou, Theodoros P Angelopoulos and John Doupis have no financial or non-financial relationships or activities to declare in relation to this article.

Review process: Double-blind peer review. Data availability: Data sharing is not applicable to this article as no datasets were generated or analysed during the writing of this article.

Compliance with ethics: This study involves a review of the literature and did not involve any studies with human or animal subjects performed by any of the authors.

Authorship: The named authors meet the International Committee of Medical Journal Editors (ICMJE) criteria

for authorship of this manuscript, take responsibility for the integrity of the work as a whole and have given final approval for the version to be published.

Access: This article is freely accessible at touchENDOCRINOLOGY.com (c) Touch Medical Media 2021

Received: 2 July 2020

Accepted: 29 June 2021

Published online: 17 November 2021

Citation: touchREVIEWS in Endocrinology. 2021;17(2):92-101

Corresponding author: John Doupis, Department of Internal Medicine and Diabetes, Salamis Naval and Veterans Hospital, Salamis Naval Base, 18900, Salamis, Attiki, Greece. E: john.doupis@harvard-alumni.org

Support: No funding was received for

the publication of this article.
Diabetes mellitus (DM) is a chronic metabolic disorder characterized by hyperglycaemia resulting from insulin resistance, inefficient insulin secretion and disproportionate glucagon secretion. ${ }^{1}$ It has been reported to be an expanding global health issue of the 21st century, and one of the leading causes of morbidity and mortality in Western countries. ${ }^{2,3}$

Interestingly, an increasing number of publications have revealed a significant correlation between DM, cardiovascular disease (CVD) and renal disease. Patients with diabetes and CVD eventually develop renal dysfunction, which is considered a poor prognostic factor. ${ }^{4} \mathrm{DM}$ and CVD share common pathophysiological pathways, including insulin resistance, inflammation, hypertension, oxidative stress and hypercoagulability. ${ }^{5}$ Recent studies have shown that individuals with diabetes and established CVD are at increased risk of developing chronic kidney disease compared with those without CVD. ${ }^{3}$ Thus, the combination of cardiac and renal impairment, which is correlated with haemodynamic dysfunction, is known as cardiorenal syndrome (CRS). ${ }^{1}$ In CRS, the acute or chronic functional impairment of one of these organs can lead to the impairment of the other., Furthermore, the initial belief regarding the primary process involved in CRS pathogenesis was the decreased cardiac pump capacity with increased fluid retention. However, different pathways influence the progression of CRS pathogenesis, including neurohormonal imbalance, endothelial damage, inflammation and atherosclerosis. ${ }^{6,7}$

The therapeutic approach for CRS is mostly centred on haemodynamic regulation. ${ }^{6}$ The main drug categories include vasodilators, diuretics, arginine vasopressin (AVP) antagonists and inotropes. ${ }^{8}$ However, many patients will eventually develop resistance to drugs such as inotropes and diuretics. Thus, an increasing number of studies have focused on investigating new therapeutic strategies to overcome such obstacles. ${ }^{8}$

This article aims to describe CRS pathogenesis, summarize the results of most current cardiovascular outcomes trials (CVOT) and review the novel therapeutic strategies. ${ }^{\text {? }}$

\section{The pathophysiology of cardiorenal syndrome}

CRS pathogenesis is based on the reciprocal interaction between the kidneys and the heart, and the effect of several other factors on this interplay. ${ }^{1}$ Specifically, the main pathophysiological pathways involved in the induction of CRS include both haemodynamic and neurohormonal mechanisms such as the sympathetic nervous system (SNS), renin-angiotensin-aldosterone system (RAAS) and AVP activation. ${ }^{1,2}$ Additionally, the inflammatory response and oxidative stress play essential roles in the initiation of cardiac, renal and endothelial injury (Figure 1). ${ }^{2}$ 


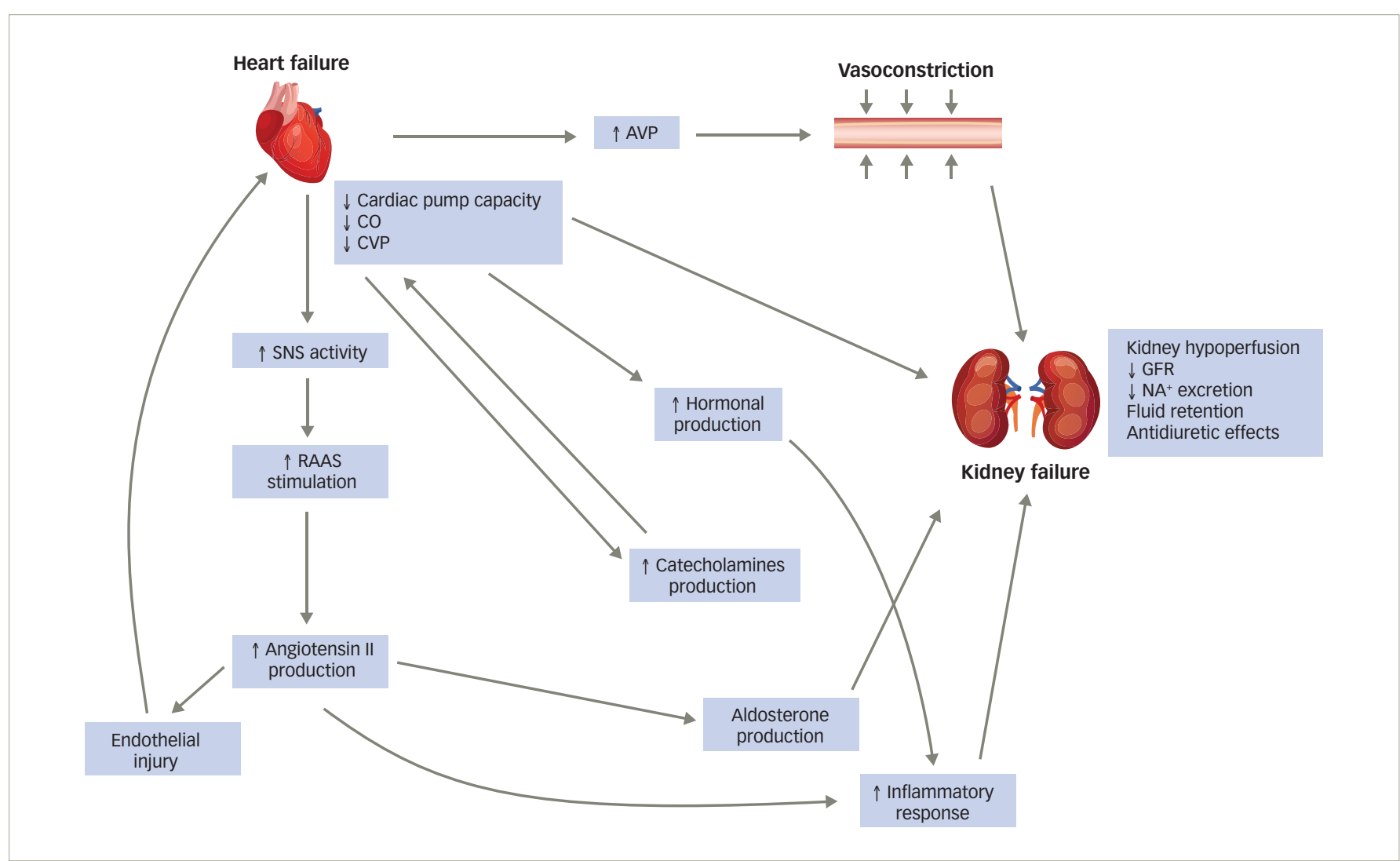

AVP = arginine vasopressin; $C O=$ cardiac output $; C V P=$ central venous pressure; $G F R=$ glomerular filtration rate; $N A=$ sodium; $R A A S=$ renin-angiotensin-aldosterone system; SNS = sympathetic nervous system.

First, concerning the haemodynamic mechanisms involved in CRS, it was found that left ventricular dysfunction represents the initial step in CRS pathogenesis. This eventually results in renal hypoperfusion, which then contributes to the initiation of the fluid retention cascade. Consequently, the cardiac pump capacity deteriorates, leading to a chain reaction and finally multiple organ dysfunction., ${ }^{1,2}$ However, although the abovementioned mechanism is fundamental for CRS pathogenesis, recent studies found that this interaction between cardiac and renal failure can occur even in patients with heart failure (HF) and preserved ejection fraction. Moreover, increased central venous pressure is an essential factor of kidney dysfunction and eventually causes a reduction in both glomerular filtration rate (GFR) and renal sodium excretion. ${ }^{1}$ Furthermore, the haemodynamic mechanism of CRS is influenced by the elevated concentration of plasma AVP in individuals with HF. The increased AVP levels provoke vasoconstriction by binding to vasopressin V1 receptors, followed by afterload elevation and fluid retention through vasopressin V2 receptor stimulation. ${ }^{1,2}$

The hyperactivation of the SNS has detrimental consequences in patients with $\mathrm{CRS}$. In HF, the continuous increased adrenergic activity causes a decrease in $\beta 1$-adrenergic receptors of the myocardium and thus an uneven $\beta 1$ to $\beta 2$ receptor ratio. This ultimately leads to a defective signal transduction cascade. However, the influence of this increased SNS activity on renal function remains unclear. Nevertheless, cardiac and renal deterioration can be induced by increased renal adrenergic activity, extensive production of catecholamines and reduced catecholamines elimination. ${ }^{1,2}$

Renal hypoperfusion triggers the release of renin, leading to activation of both the RAAS and the SNS. ${ }^{2}$ Subsequent vasoconstriction, the rise of afterload and, consequently, the reduction of cardiac output constitute another major cascade mechanism in CRS progression. ${ }^{1}$ Specifically, a significant detrimental effect of RAAS is the stimulation and production of nicotinamide adenine dinucleotide phosphate (NADPH) oxidase, followed by the release of reactive oxygen species. ${ }^{2}$ Furthermore, angiotensin II production induces the activation of an inflammatory cascade, which eventually leads to apoptosis, fibrosis, endothelial damage and cardiovascular (CV) hypertrophy. In particular, angiotensin II is associated with a rise in the synthesis of renal tumour necrosis factor, nuclear factor $\kappa B$, interleukin 6 and monocyte chemoattractant protein 1. Moreover, it also induces superoxide production via stimulation of nicotinamide adenine dinucleotide and NADPH oxidase, which are both associated with renal failure and HF.1,2 Furthermore, angiotensin II stimulates the release of aldosterone, which binds to myocardial mineralocorticoid receptors and exerts important effects on endothelial injury, HF and hypertrophy. ${ }^{1}$

Therefore, the standard therapeutic management of patients with DM presenting with cardiorenal comorbidities includes angiotensin-converting enzyme inhibitors and angiotensin receptor blockers. ${ }^{4}$ This treatment approach has proven to exert significant cardiorenal protection in patients with $\mathrm{HF}_{\text {, }}$ and diabetic and nondiabetic nephropathy via RAAS and SNS blockade. ${ }^{2}$ In particular, angiotensin-converting enzyme inhibitors have both antiproteinuric and antihypertensive functions, which decelerate the renal damage in individuals with type 1 diabetes mellitus (T1DM). Similarly, these drugs can delay the progression of diabetic nephropathy in patients with type 2 diabetes mellitus (T2DM). Moreover, studies found that patients with T2DM with or without diagnosed diabetic nephropathy treated with angiotensin receptor blockers showed less proteinuria, and lower rates of disease progression and dialysis. Remarkably, inhibition of RAAS 
decreases $\mathrm{CV}$ death rates in patients who are at high risk according to the European Society of Cardiology (ESC) Systematic Coronary Risk Evaluation (SCORE) Risk Charts. ${ }^{4}$

In patients with heart and renal failure, endothelial dysfunction is an important factor of impaired vasomotor activity.1,2 Endothelial injury is associated with reduced nitric oxide activity and elevated reactive oxygen species production, which finally causes hyperactivity of SNS., ${ }^{2,3}$ Finally, adenosine was found to modulate the renal function via the A1 receptor, which subsequently causes GFR reduction, and a rise in sodium absorption and antidiuretic action. In addition, increased plasma adenosine concentration has been found in patients with HF during hypoxia states. In conclusion, adenosine is considered a significant contributor in the CRS pathogenesis. ${ }^{1,2}$

The management and treatment of patients with CRS have been demanding for healthcare professionals owing to the complicated pathophysiology of this syndrome. ${ }^{5}$ Over the years, studies proved an elevated CV risk associated with the use of certain antiglycaemic drugs. Consequently, numerous clinical trials were conducted and eventually, novel classes of antidiabetic therapies with established CV safety emerged, such as glucagon-like sodium-glucose cotransporter-2 (SGLT-2) inhibitors, peptide-1 (GLP-1) receptor agonists and dipeptidyl peptidase-4 (DPP-4) inhibitors. ${ }^{6}$ SGLT-2 inhibitors exert several beneficial effects on renal function, including the decrease of intraglomerular hypertension, oxidative stress, glomerular hyperfiltration, glomerular inflammatory and fibrotic response..$^{-9}$ Similar cardiorenal effects have been reported following treatment with GLP-1 agonists. ${ }^{10}$

\section{The future of treating diabetes as a cardiorenal-metabolic disease}

The treatment of T2DM as a cardiorenal-metabolic disease has been thoroughly studied. In the literature, several publications have reported the importance of controlling the blood glucose levels, blood pressure and dyslipidaemia in diabetic individuals. ${ }^{11}$ Subsequently, numerous metabolic pathways have been investigated in order to develop new antidiabetic therapies. However, further research is expected in this specific area.

The incretin system is currently an important pathway target for the management of T2DM. ${ }^{11}$ There are two main classes of incretin-related therapies: GLP-1 analogues and DPP-4 inhibitors. ${ }^{11-13}$ GLP-1 agonists affect glucose metabolism in the same way as endogenous incretins. Exenatide, liraglutide, dulaglutide, semaglutide and albiglutide are the five primary GLP-1 analogues. The mechanisms of action of these agents include the increase of insulin secretion, the reduction of postprandial glucagon concentration and the deceleration of the gastric emptying process. ${ }^{12}$ The results of recent studies have shown that these drugs improve blood glucose levels, along with a notable reduction in body weight and systolic blood pressure. ${ }^{11-13}$ Additionally, a decrease of albuminuria levels was also observed in several uncontrolled studies. Of interest, liraglutide is one of the few approved drugs (other approved drugs of the same class include dulaglutide and semaglutide), that have been established by the US Food and Drug Administration (FDA) for decreasing the risk of CV events in patients with known T2DM and CVD. ${ }^{12}$ Remarkably, various studies such as the LEADER and SUSTAIN-6 trials revealed a reduction in nephropathy development and progression (please see full trial names and clinical trials details in Table 1). ${ }^{11}$

In the HARMONY trial, Iow rates of the primary outcomes (CV death, non-fatal myocardial infarction (MI), non-fatal stroke) were demonstrated. ${ }^{11}$ The cardiorenal protective mechanisms of these drugs include decreased inflammatory response, antioxidant and antifibrotic actions. ${ }^{11,14}$ Additionally, GLP-1 agonists demonstrated a preventive role in the new onset of albuminuria, which is achieved by regulating the blood glucose levels, enhancing natriuresis, and decreasing systolic blood pressure and body mass index. ${ }^{15}$

The most frequent adverse events reported following treatment with GLP-1 receptor agonists are nausea and vomiting, especially with short-acting agonists. However, the side effects of this treatment are mostly temporary. In the LEADER trial, a high percentage of patients developed gallstones and acute cholecystitis. The SUSTAIN-6 trial outcomes showed a high rate of retinopathy in patients treated with a GLP-1 agonist. Finally, the results of the FIGHT14 trial revealed that patients with HF had high rates of readmission following the use of GLP-1 agonists. ${ }^{14,16}$

DPP-4 inhibitors act by inhibiting the DPP-4 enzyme, leading to a rise of GLP-1, along with an increase of insulin levels and simultaneous inhibition of glucagon secretion. ${ }^{11,13}$ DPP-4 inhibitors include saxagliptin, sitagliptin, alogliptin, vildagliptin and linagliptin. ${ }^{11,15}$ Linagliptin is the most well-studied agent used in patients with known diabetic nephropathy. The LIRA-RENAL12 randomized controlled trial demonstrated that patients on linagliptin presented low levels of albuminuria and glycated haemoglobin A1C (HDA1C). 12,17 Remarkably, in the SAVOR-TIMI 53, TECOS, VERIFY and EXAMINE trials, the patients treated with saxagliptin, sitagliptin, vildagliptin and alogliptin presented with almost the same CVD outcomes as the placebo group..$^{11,18,19}$ Recent publications highlight that the inhibition of the DPP-4 enzyme exhibits many cardiorenal benefits through blood glucose levels and body weight reduction. Further cardiorenal protective mechanisms of these drugs include the decrease of inflammatory response and oxidative stress, as well as their antifibrotic actions. ${ }^{11}$

Another novel hypoglycaemic drug class is SGLT-2 inhibitors, which include dapagliflozin, empagliflozin, canagliflozin, sotagliflozin and ertugliflozin..$^{11,20}$ The cardiorenal benefits of SGLT-2 inhibitors were noticed following the progress of six major trials: CANVAS, EMPA-REG OUTCOME, DECLARE-TIMI 58, SCORED, SOLOIST-WHF and VERTIS. ${ }^{21}$

SGLT-2 is a cotransporter of sodium and glucose, detected in the proximal tubule of the nephron. ${ }^{21}$ It reabsorbs almost $90 \%$ of the urinary glucose. Notably, a significant glucose hyperfiltration has been observed in individuals with DM, which eventually stimulates the activation of SGLT-2 receptors, resulting in systemic hyperglycaemia, blood pressure dysregulation and hypernatraemia. ${ }^{14,21}$ The mechanism of action of SGLT-2 inhibitors is based on the inhibition of SGLT-2 in the proximal convoluted tubule of the kidney. ${ }^{12}$ This mechanism of drug action leads to a decrease of renal glucose reabsorption, which causes glycosuria and eventually reduces the blood glucose levels. ${ }^{11,15}$ The reduction of blood glucose levels discarded through urine is achieved in an insulin-independent process. Thus, these drugs cause a decrease in blood glucose concentration and body weight, while preventing the possibility of hypoglycaemia. ${ }^{14}$ Another essential cardiorenal protective mechanism of this therapy is the enhancement of natriuresis and the subsequent decrease of hyperfiltration, resulting in blood pressure reduction. Finally, even though it is considered debatable, SGLT-2 inhibitors have a direct cardioprotective effect, which is achieved through blockade of cardiac SGLT-2 receptors. ${ }^{21}$

Concerning the safety profile of the SGLT-2 inhibitors, the results of a recent meta-analysis have shown a link between the use of these drugs and an increased rate of genital and urinary tract infections. ${ }^{21}$ Moreover, 
Table 1: Cardiovascular outcome trials on type 2 diabetes

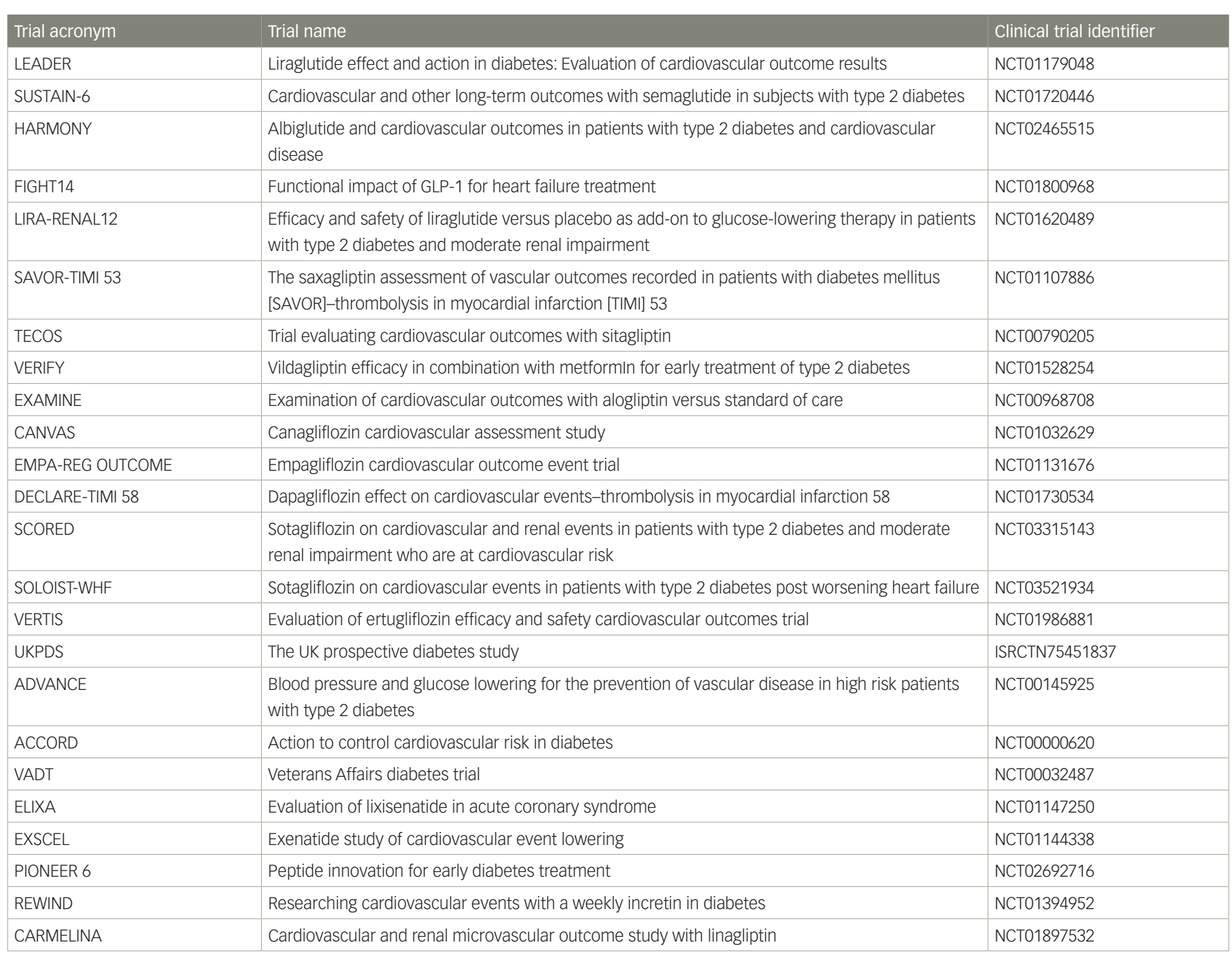

low rates of diabetic ketoacidosis have been reported following the use of SGLT-2 inhibitors. ${ }^{14}$ Other side effects of SGLT-2 inhibitors are lower extremity amputations, bone fractures and urinary bladder malignancy. Interestingly, current data reveal an increased risk of Fourier gangrene following treatment with these drugs. Overall, the commonest and most reported side effect is genital area infections. ${ }^{21}$

Recently, many attempts have been made to develop novel antidiabetic drugs. Endothelin-1 receptor antagonists are a new class of antidiabetic drugs that prevent albuminuria. An on-going trial was, however, terminated early because of adverse outcomes in the treatment group (i.e. fluid retention and risk of congestive HF). Currently, there is considerable interest in the investigation of drugs that may promote autophagy. Finally, Src family kinases consist of a new treatment goal for the management of T2DM. ${ }^{11}$

\section{The rationale for treating type 2 diabetes: Analysing the UKPDS, ACCORD, ADVANCE and VADT trials}

UKPDS was the first study to examine the correlation of tight blood glucose control with morbidity and mortality in patients newly diagnosed with T2DM. The purpose of this trial was to investigate whether treating patients with T2DM with the currently available antidiabetic medications could concurrently ultimately provide micro- and macrovascular protection against the detrimental effect of DM on vascular integrity. ${ }^{22}$ The sample comprised 5,100 patients newly diagnosed with T2DM. The median age was 53 years and median therapy duration was 11 years. The patients receiving intensive blood glucose control achieved a median $\mathrm{HbA} 1 \mathrm{C}$ of $7.0 \%$, compared with $7.9 \%$ in patients under less tight blood glucose control. Previous clinical trials demonstrated a correlation between euglycaemic levels in patients with DM, and a decline in morbidity and mortality due to CV events. However, UKPDS demonstrated undoubtedly that even with a $1 \%$ reduction in HbA1C, microvascular complications were decreased by $37 \%$, yet no statistically significant decrease in macrovascular complications was noted.22,23 Building upon the findings of the UKPDS trial, three additional trials took the lead, addressing the question of whether strict blood glucose levels could positively alter the prognosis of CVD outcomes in high-risk patients with T2DM.

First, the ADVANCE trial randomized 11,140 patients with T2DM from 215 centres in 20 countries around the world. Patients were aged $>55$ years and had at least one risk factor for a CV event. The primary outcomes were any of the three-point major adverse CV events (3-P MACE). Overall, the primary endpoint was met in 2,125 patients, $18.1 \%$ in the intensive group and $20.0 \%$ in the standard group. The overall analysis showed that there were no significant differences between the two groups with regard to macrovascular events. ${ }^{23}$ Similarly, the 
ACCORD trial served to answer whether a much stricter $\mathrm{HbA} 1 \mathrm{C}<6.0 \%$ could offer additional CV protection. This trial included 10,521 patients who were randomly assigned into an intensive glycaemic control group and a standard therapy group. The primary outcomes were a composite of 3-P MACE, and the mean follow-up was 3.5 years. Although the primary outcomes were reduced in the intensive glycaemic control group, the difference was statistically insignificant; however, surprisingly, an increased rate of mortality was noted, leading to the early termination of the trial. ${ }^{23,24}$

Overall, all three trials failed to show a significant reduction in adverse CV outcomes in patients with T2DM. The parameters that demonstrate prevention of adverse events in patients with diabetes are more complicated than in patients without diabetes, contributing to the difficulty of demonstrating CV protection in patients with diabetes. It is estimated that the strategies adopted in the three trials failed to provide significant reductions in adverse CV events, partly due to the pure focus on the potency of the antiglycaemic drugs, while the side effects could counteract the efforts for $\mathrm{CV}$ protection..$^{24}$

The VADT trial was designed to study the result of intensive antidiabetic therapy compared with standard therapy on CV outcomes in patients with T2DM. In the VADT trial, 1,791 patients with DM were randomized to either intensive antiglycaemic therapy (maximum therapeutic doses, aim: $\mathrm{HbA} 1 \mathrm{C}<6.0 \%$ ) or standard therapy (half of the maximum therapeutic doses, aim: $\mathrm{HbA} 1 \mathrm{c}$ reduction of at least $1.5 \%$ ). The mean patient age was 60 years, the average duration of DM was 11.5 years and the average level of HbA1C was $9.4 \%$. Approximately $75 \%$ of patients presented with hypertension, and $40 \%$ had experienced a prior CV event. ${ }^{25}$ Many antidiabetic drugs such as rosiglitazone, metformin, insulin and glimepiride were used to control blood glucose levels. Two antiglycaemic agents were given in both groups, and patients achieved median $\mathrm{HbA} 1 \mathrm{C}$ values of $6.9 \%$ and $8.4 \%$ in the intensive therapy and standard therapy groups, respectively. ${ }^{25}$ The trial results found almost equal incidence of the primary endpoints (i.e. CV events including stroke, Ml, CV death, congestive HF, non-operative coronary artery disease, amputation of ischaemic origin) between the two groups. ${ }^{24,25}$ Remarkably, individuals treated in the intensive antiglycaemic therapy group had slightly increased mortality rates due to CVD events than those treated with standard therapy. ${ }^{24}$ Furthermore, hypoglycaemia was more common in the intensive therapy group (8.5\%) than in the standard therapy group (3.1\%). ${ }^{25}$ Finally, patients who had DM for $<12$ years showed a more favourable CVD outcome following intensive antidiabetic treatment than those with DM of longer duration, who presented a more unfavourable outcome. ${ }^{24}$

\section{New cardiovascular outcome trials in diabetes}

In 2007, a meta-analysis of randomized controlled trials of rosiglitazone concluded that there was an increased risk of $\mathrm{Ml}$ and death in individuals receiving rosiglitazone. This triggered a series of discussions regarding the need to evaluate antidiabetic therapies from a CV perspective. In December 2008, the FDA provided 'Guidance for Industry' stating the importance of evaluating every new antihyperglycaemic drug for T2DM in order to prevent any undesirable CV outcomes due to antidiabetic therapy. ${ }^{26,27}$ As a result, a large number of CVOTs were conducted to evaluate all new glycaemic control therapies from a CV perspective, examining a broad spectrum of CV outcomes. ${ }^{26,28}$ The primary CV endpoint was the 3-P MACE, comprising 1) CV mortality, 2) non-fatal MI and 3) non-fatal stroke..$^{20}$ Additionally, in secondary CV outcomes, all-cause mortality, hospitalization due to $\mathrm{HF}$ and renal outcomes were included. ${ }^{26}$
To date, 17 CVOTs have been completed including medication from four main antiglycaemic drug categories: 1) DPP-4 inhibitors, 2) GLP-1 receptor agonists, 3) SGLT-2 inhibitors and 4) insulin (glargine, degludec). ${ }^{28,29}$

\section{Dipeptidyl peptidase-4 inhibitor cardiovascular outcome trials}

The TECOS study was a double-blind, randomized, phase III clinical trial designed to examine the CV outcomes of sitagliptin in patients with diabetes, established CVD and an HBA1c between 6.5\% and $8.0 \%$ (48-64 mmol/mol) under a constant dose of glucose-lowering drugs. ${ }^{30} \mathrm{~A}$ total of 14,735 participants were enrolled in the trial and randomized to receive either sitagliptin or placebo. ${ }^{31}$ The results of a 3-year follow-up period showed that $11.4 \%$ of the sitagliptin-treated group (851 patients) presented primary 3-P MACE events, compared with $11.6 \%$ in the control group (851 patients). ${ }^{32}$ Additionally, the TECOS trial results showed neutral findings regarding the secondary outcomes such as non-fatal stroke events and hospitalization for $\mathrm{HF}^{32}$

Moreover, the CV profile of saxagliptin in patients with DM was examined in the SAVOR-TIMI 53 trial. This was a double-blind, placebo-controlled, randomized trial with a total of 16,492 patients and a median follow-up duration of 2 years. ${ }^{33,34}$ The initial purpose was to examine the CV effects of saxagliptin in patients with diabetes either at high risk for CV events or with an already established CVD. ${ }^{33,34}$ Patients with diabetes and high $\mathrm{CV}$ risk were classified based on kidney function, defined as normal, mild or severe renal deterioration, and then randomized further into a saxagliptin-treated group or a placebo group..$^{35}$ The primary endpoint of SAVOR-TIMI 53 was 3-P MACE. ${ }^{33,34}$ Results in the saxagliptin-treated group were similar to those of the control group, regardless of renal function. .3,35 $^{3.3}$ specifically, primary outcome events were observed in $7.3 \%$ of patients in the saxagliptin group and $7.2 \%$ in the control group. ${ }^{34}$ Secondary outcomes (CV mortality, Ml, stroke, unstable angina, hospitalization for $\mathrm{HF}$, and coronary revascularization) were almost equivalent between the two groups, and occurred in $12.8 \%$ of the intervention group and $12.4 \%$ of the control group. Nevertheless, more hospitalizations for HF were observed in the saxagliptin group compared with the control group (3.5\% versus $2.8 \% ; \mathrm{p}=0.007$ ). ${ }^{34}$ Overall, the SAVOR-TIMI 53 trial highlighted that although saxagliptin is unrelated to an increase in the risk of ischaemic events, a correlation between saxagliptin and increased rate of hospitalization for HF does exist. ${ }^{36}$

The CARMELINA trial was designed to examine the cardiorenal effects of linagliptin in patients with T2DM at high risk for CVD and with established renal dysfunction. ${ }^{37}$ CARMELINA was a randomized, double-blind, placebo-controlled trial involving 6,991 patients who were selected based on specific inclusion criteria, including established T2DM, high $\mathrm{CV}$ risk, renal dysfunction and HbA1c levels ranging between $6.5 \%$ and $10.0 \% .{ }^{37-39}$ In this study, 3,494 patients were randomized to the linagliptin-treated group and 3,485 to the placebo group. ${ }^{37}$ The primary outcome was time to first incidence of 3-P MACE, and secondary outcomes included time to first incidence of mortality due to renal impairment, end-stage renal disease or estimated GFR decline of at least $40 \% .{ }^{37,38}$ Results showed that 434 (12.4\%) of the linagliptin-treated patients presented with 3-P MACE, compared with 420 patients (12.1\%) in the placebo group. ${ }^{37}$ Overall, the incidence variance between the two groups was 0.13 per 100 person-years, revealing that linagliptin was not superior to placebo. ${ }^{37}$ However, linagliptin did not appear to increase the risk of CV events compared with placebo. Finally, secondary renal outcomes were noticed in $9.4 \%$ of patients in the linagliptin group and $8.8 \%$ of patients in the placebo group. ${ }^{39}$ 
The VERIFY trial compared metformin monotherapy with combination therapy with vildagliptin plus metformin in patients newly diagnosed with T2DM and at low risk for CVD.40 VERIFY was a randomized, double-blind, placebo-controlled trial in which a total of 2,001 patients were enrolled. The study focused on glycaemic control, comparing the efficacy of the two treatments. Although the trial was not powered or designed to assess the effect of vildagliptin treatment on CV outcomes, an imbalance was noticed favouring combination therapy. CV death, non-fatal Ml or stroke and hospital admission for HF were included as a composite of $\mathrm{CV}$ outcomes and were described as exploratory outcomes. The results showed that an event occurred in $24(2.4 \%)$ of the 998 patients in the combination group, compared with 33 (3.3\%) of the 1,001 patients in the monotherapy group (hazard ratio [HR] 0.71; $95 \%$ confidence interval $[\mathrm{Cl}], 0.42-1.19$; two-sided $\mathrm{p}=0.19$ ). The major limitation of this study was that it was not powered to assess the effect of vildagliptin on CV outcomes, but all potential CV events were subject to adjudication. Overall, the results from this trial indicate a difference in CV events in favour of vildagliptin, which was not significant, and focus on patients with low CV risk. ${ }^{41}$

\section{Glucagon-like peptide 1 receptor agonist cardiovascular outcome trials}

The purpose of the ELIXA trial was to examine the CV effects of lixisenatide, a GLP-1 receptor agonist, in patients with diabetes at high $\mathrm{CV}$ risk who had a recent acute coronary event. ${ }^{42-44}$ ELIXA was a placebo-controlled, double-blind, phase III, randomized trial with a total of 6,068 patients. ${ }^{42-44}$ Primary outcomes (i.e. CV mortality causes, non-fatal MI, non-fatal stroke or hospitalization for unstable angina) were observed in $13.4 \%$ of the lixisenatide-treated group compared with $13.2 \%$ in the control group. ${ }^{43}$ Further analysis revealed that patients with diabetes and previous acute coronary syndrome events who received lixisenatide had similar CV outcomes compared with the placebo group. ${ }^{43}$ Additionally, insignificant variations were noted in secondary outcomes (i.e. hospitalization for HF, coronary revascularization) among the two groups. The ELIXA study concluded that lixisenatide was non-inferior, but also non-superior to placebo in the protection against adverse $\mathrm{CV}$ outcomes. ${ }^{42}$

The LEADER study was a double-blind trial in 9,340 patients (mean age 64 years) who were randomized into two subgroups, in order to test the non-inferiority of liraglutide to placebo treatment with regard to CV outcomes (composite outcome 3-P MACE). ${ }^{45}$ One group received liraglutide $(n=4,668)$ and the other placebo $(n=4,672)$, both in addition to standard care. High CV risk was defined as multiple risk factors for CVD or as established CVD. ${ }^{46}$ Median follow-up time was 3.8 years. Overall, there was a lower incidence of the composite endpoints in the group treated with liraglutide $(608 / 4,668 ; 13.0 \%)$ than in the placebo group $(694 / 4,672 ; 14.9 \%)(H R, 0.87 ; 95 \% \mathrm{Cl}, 0.78-0.97 ; p<0.001$ for non-inferiority; $\mathrm{p}=0.01$ for superiority). ${ }^{47}$ Moreover, the rate of death from any cause was significantly lower in the liraglutide group (381 patients [8.2\%]) than in the placebo group (447 [9.6\%]) (HR, 0.85; 95\% $\mathrm{Cl}, 0.74-0.97 ; \mathrm{p}=0.02$ ). LEADER proved that liraglutide has major direct and indirect protective effects on the CV system in patients with T2DM and high CV risk index, while also playing a role in lowering the incidence of the commonest CV events compared with placebo, even with the addition of other antidiabetic agents. In addition, liraglutide proved to maintain blood glucose levels and significantly impact the long-term preservation of euglycaemia in patients with T2DM.

The aim of the EXSCEL study was to show that exenatide was non-inferior to placebo regarding CV safety, investigating possible additional positive $\mathrm{CV}$ effects in patients with T2DM. ${ }^{48,49}$ The study was conducted at 687 sites in 35 countries, and included a total of 14,752 patients with T2DM, 70\% of whom had a history of established CVD. The patients were randomized to treatment with either exenatide or placebo. The primary composite outcome was any first incidence of the 3-P MACE. The mean follow-up was 3.2 years. Primary outcome events were observed in 839 of 7,356 patients (11.4\%) in the exenatide group and in 905 of 7,396 patients (12.2\%) in the placebo group. The exenatide group was observed to have better CV outcomes, with fewer primary 3-P MACE endpoints compared with placebo; however, the statistical analysis showed that the difference was not significant. The trial had a major limitation, which was the premature discontinuation of the regimen due to patient decision. In conclusion, it was proven that exenatide was non-inferior to placebo with regard to safety, but non-superior in terms of efficacy. ${ }^{48,49}$

The SUSTAIN-6 trial was conducted to investigate the non-inferiority of subcutaneous semaglutide, compared with placebo, in patients with T2DM and high CV risk. The primary composite outcome was 3-P MACE. It was a randomized, double-blind, placebo-controlled, parallel-group trial conducted at 230 sites in 20 countries, and included a total of 3,297 patients. ${ }^{50,51}$ The mean follow-up time was 2.1 years. The trial showed that the primary composite outcome of 3-P MACE was met in $6.6 \%$ of the semaglutide group (108/1,648 patients) and in $8.9 \%$ of the placebo group (146/1,649 patients). Overall, the patients treated with semaglutide had a $26 \%$ less risk of meeting a primary composite outcome than those treated with placebo. A significant 39\% decrease in stroke, as well as a $26 \%$ decrease of non-fatal MI, contributed to the results. ${ }^{50-52}$ Concurrently, based on albuminuria levels, patients treated with semaglutide exhibited a lower risk for new-onset or worsening nephropathy when compared with the placebo group. ${ }^{51,52}$ Concerning side effects, retinopathy complications were significantly higher in the semaglutide group. Although serious adverse events were lower in the semaglutide group, more patients discontinued their regimen because of adverse events, which were mainly gastrointestinal in nature. The study was designed to examine non-inferiority over a placebo, yet the $\mathrm{CV}$ - and reno-protective profile of semaglutide was evident. ${ }^{50,51}$

An additional study was conducted using oral semaglutide in patients with T2DM at high CV risk, with the same primary endpoint of 3-P MACE. ${ }^{53}$ PIONEER 6 was a double-blind, randomized, placebo-controlled trial in which $85 \%$ of patients were older than 50 years and had CVD or chronic kidney disease. The median follow-up time was 15.9 months. This trial also showed no inferiority of semaglutide compared with placebo (HR, $0.79 ; 95 \% \mathrm{Cl}, 0.57-1.11)$. It is also worth mentioning that death from CV causes and all-cause death were significantly different between the two groups, in favour of semaglutide. ${ }^{53}$

Another important study was the HARMONY trial, which was conducted to investigate the efficacy and safety of albiglutide compared with placebo for 3-P MACE in patients with T2DM and established CVD. The trial was a double-blind, randomized, placebo-controlled trial and included 9,463 randomized patients. The median follow-up was approximately 18 months. The composite endpoint of 3-P MACE occurred in 338 patients (7\%) in the albiglutide group and 428 patients $(9 \%)$ in the placebo group ( $\mathrm{HR}, 0.78 ; 95 \% \mathrm{Cl}, 0.68-0.90)$. It was shown that albiglutide was superior to placebo ( $\mathrm{p}=0.0006$ for superiority) in terms of major adverse CV events. Furthermore, the incidence of other serious adverse events, such as acute pancreatitis and pancreatic cancer, did not differ between the two groups. The main concern for this study was the short duration of follow up. Finally, the results of the 
HARMONY trial indicated that specific GLP-1 agonists can be useful in reducing $\mathrm{CV}$ events in patients with $\mathrm{T} 2 \mathrm{DM}{ }^{54}$

Subcutaneous dulaglutide, which is also a GLP-1 agonist, was assessed in the REWIND trial. ${ }^{55}$ This was also a randomized, double-blind, placebo-controlled trial, with 3-P MACE as the primary endpoint. The study population was patients with T2DM at high CV risk and with high HbA1C concentration. Over a median follow-up of 5.4 years, a significant difference in the primary endpoint was indicated between the two groups, favouring dulaglutide. All-cause mortality and CV mortality did not differ between the two groups. A major limitation was that $25 \%$ of patients were not taking the study drug at the time of their last visit. Overall, dulaglutide could be beneficial for patients with T2DM and CV risk to reduce glucose concentration and CV events. ${ }^{55}$

\section{Sodium-glucose cotransporter 2 inhibitors cardiovascular outcome trials}

The EMPA-REG OUTCOME study was the first CVOT that showed a significant decrease in CV events and an increase in survival rates in patients with a previous CVD history. ${ }^{55-58} \mathrm{~A}$ total of 7,020 patients with known T2DM and CVD, HbA1C levels ranging between $7 \%$ and $10 \%$, and GFR $\geq 30$ were enrolled in the study, while $65 \%$ of the patients had a history of stroke or $\mathrm{Ml}$ and, based on the thrombolysis in myocardial infarction (TIMI) risk score, $12 \%, 40 \%, 30 \%$ and $18 \%$ of them presented low, intermediate, high and very high risk, respectively. ${ }^{57}$ There was a $14 \%$ reduction in the primary composite outcome (3P-MACE) in patients who received empagliflozin, and a considerable decrease of $38 \%$ in CV mortality, along with a $32 \%$ decrease in all-cause mortality. Furthermore, a 35\% reduction in hospitalization for HF was reported compared with the placebo group..$^{58}$ Undoubtedly, the results of the trial highlighted the superiority of empagliflozin over placebo, demonstrating a $38 \%$ decrease in macroalbuminuria, $44 \%$ decrease in creatinine levels and 39\% decrease in nephropathy progression, thus reducing the incidence of renal adverse outcomes, compared with placebo. ${ }^{46,58}$ Moreover, in patients with established chronic kidney disease, treatment with empagliflozin led to a $29 \%$ decline in CV mortality, $39 \%$ lower hospitalizations due to HF and $24 \%$ fewer incidents of all-cause mortality, proving that empagliflozin has cardiorenal protective properties in patients with DM. ${ }^{57}$

The CANVAS programme was a randomized, double-blind, placebo-controlled trial designed to evaluate the CV effectiveness and safety of canagliflozin in patients with T2DM at high CV risk, with 3-P MACE as the primary endpoint. ${ }^{60,61}$ Patients were selected based on specific inclusion criteria, including established uncontrolled T2DM (defined as $\mathrm{HbA} 1 \geq 7.0 \%$ and $\leq 10.5 \%$ ), CVD and CV risk factors. ${ }^{62} \mathrm{~A}$ total of 10,142 patients were enrolled and randomized to either the canagliflozin-treated group $(n=5,795)$ or a placebo group $(n=4,347) .60,61$ Mean follow-up was 188 weeks. ${ }^{57}$ Initial analysis of the results revealed that in the canagliflozin-treated group, the incidence of any of the 3-P MACE outcomes was lower compared with the placebo group. Specifically, primary outcome events occurred in $26.9 \%$ per 1,000 patient-years in patients treated with canagliflozin and in 31.5\% per 1,000 patient-years in the placebo group. Moreover, canagliflozin showed a $33 \%$ reduction in hospitalization due to HF compared with the placebo. ${ }^{60,62,63}$ Remarkably, canagliflozin was reported to be more efficacious in individuals with a history of $\mathrm{HF}$, body mass index $\geq 30 \mathrm{~kg} / \mathrm{m}^{2}$ and $\mathrm{HbA} 1 \mathrm{C} \geq 8 \%$. Canagliflozin proved to have a potent renoprotective profile by reducing the presence or development of albuminuria incidents by $27 \%$ and $20 \%$, respectively. The limitations of the trial included the risk of false-positive results owing to the large number of analyses and relatively few events. In addition, the small proportion of patients with kidney disease limits the study's ability to generalize the results to this patient population. In general, canagliflozin exhibited both $\mathrm{CV}$ and renal benefits in patients with T2DM and established CVD or CV risk factors. ${ }^{60}$

The DECLARE-TIMI 58 study was a randomized, double-blind, multinational, placebo-controlled, phase III trial that was designed to demonstrate the non-inferiority of the SGLT-2 inhibitor dapagliflozin over placebo in patients with T2DM and established CVD or with multiple risk factors for major adverse CV events.44,65 A total of 17,160 patients aged $>40$ years and with a mean $\mathrm{HbA} 1 \mathrm{C}$ ranging from $6.5 \%$ to $12 \%$, were randomized to receive dapagliflozin or placebo. Of the patients enrolled, 6,974 (40.6\%) had a profound atherosclerotic disease, whereas the majority (10,186 patients; $59.4 \%)$ had risk factors for atherosclerotic disease. Mean follow-up was 4.2 years. The study showed that patients treated with dapagliflozin presented non-inferior results in major CV events compared with the placebo group. The rates of deaths or hospitalizations due to HF were significantly lower in the dapagliflozin group (4.9\%) compared with the placebo group (5.8\%; HR, 0.83; $95 \%$ $\mathrm{Cl}, 0.73-0.95 ; \mathrm{p}=0.005)$, whereas the rate of a 3-P MACE was not lower compared with patients treated with placebo. Furthermore, each of the subgroups of patients with T2DM, based on the presence or absence of established atherosclerotic CVD, benefitted from dapagliflozin in terms of HF; patients with established atherosclerosis had a lower rate of hospitalization for HF and death, while for patients with multiple risk factors for atherosclerosis, HF events deriving from atherosclerotic disease could be prevented early. However, urinary infections and diabetic ketoacidosis were more common in the dapagliflozin group compared with the placebo group. In this trial, patients with chronic kidney disease, who derive great benefit from SGLT-2 inhibitors, were excluded; the exclusion of these patients probably affected the mortality benefit of SGLT-2 inhibitors in the study. ${ }^{64}$

VERTIS was a randomized, double-blind, multicentre trial aimed at assessing the efficacy and safety of ertugliflozin compared with placebo. ${ }^{66}$ The subjects of this trial were patients with T2DM and established atherosclerotic CVD above the age of 40 years. Exclusion criteria were T1DM or a history of diabetic ketoacidosis and GFR below $30 \mathrm{~mL} / \mathrm{min} / 1.73 \mathrm{~m}^{2}$. A total of 8,246 patients were randomized to ertugliflozin or placebo. The primary outcome was a composite of 3-P MACE, and the secondary outcome was a composite of death from CV causes or hospitalization for HF. Mean follow-up was 3.5 years. The study showed that ertugliflozin was non-inferior to placebo for the primary outcome of 3-P MACE, with both groups having the same proportion of events (11.9\%). The secondary outcome occurred in 444 patients (8.1\%) in the ertugliflozin group and in 250 patients (9.1\%) in the placebo group ( $\mathrm{HR}, 0.88 ; 95.8 \% \mathrm{Cl}, 0.75-1.03 ; \mathrm{p}=0.11$ for superiority). The rates of serious adverse events, including fractures, diabetic ketoacidosis, urinary tract infection and acute kidney injury, did not differ significantly between the two groups. Overall, the VERTIS trial indicated that ertugliflozin was non-inferior to placebo with respect to 3-P MACE, but also non-superior in reducing the rate of $\mathrm{CV}$ deaths or hospitalizations due to $\mathrm{HF}^{.66}$

The SOLOIST-WHF trial was a randomized, double-blind trial that tested the efficacy and safety of sotagliflozin versus placebo in patients with T2DM who were recently hospitalized for worsening HF. ${ }^{67}$ Exclusion criteria included recent acute coronary syndrome, previous percutaneous coronary intervention or coronary artery bypass surgery, end-stage HF and GFR $<30 \mathrm{~mL} / \mathrm{min} / 1.73 \mathrm{~m}^{2}$. A total of 1,222 patients were randomized. The primary endpoint was the total number of deaths from CV causes, 
Table 2: Summary of cardiovascular outcome trials in type 2 diabetes

\begin{tabular}{|c|c|c|c|c|c|c|}
\hline Study & Drug & Patients, $\mathrm{n}$ & Median follow-up & $\begin{array}{l}\text { Three-point MACE, } \\
\text { HR }(95 \% \mathrm{Cl})\end{array}$ & $\begin{array}{l}\text { Hospitalization for } \\
\text { heart failure, } \\
\text { HR }(95 \% \mathrm{CI})\end{array}$ & $\begin{array}{l}\text { Renal outcomes, } \\
\text { HR }(95 \% \mathrm{Cl})\end{array}$ \\
\hline \multicolumn{7}{|c|}{ GLP-1 receptor agonist studies } \\
\hline ELIXA $^{41,43}$ & Lixisenatide & 6,068 & 108 weeks & $1.02(0.89-1.17)$ & $0.96(0.75-1.23)$ & NR \\
\hline LEADER ${ }^{46}$ & Liraglutide & 9,340 & 3.8 years & $0.87(0.78-0.97)$ & $0.87(0.73-1.05)$ & $0.78(0.67-0.92)$ \\
\hline EXSCEL $^{47}$ & Exenatide & 14,752 & 3.2 years & $0.91(0.83-1.00)$ & $0.94(0.78-1.13)$ & NR \\
\hline SUSTAIN-64,50 & Semaglutide, SC & 3,297 & 2.1 years (mean) & $0.74(0.58-0.95)$ & $1.11(0.77-1.61)$ & $0.64(0.46-0.88)$ \\
\hline PIONEER $6^{52}$ & Semaglutide, PO & 3,183 & 15.9 months & $0.79(0.57-1.11)$ & $0.86(0.48-1.55)$ & NR \\
\hline HARMONY53 & Albiglutide & 9,463 & 1.5 years & $0.78(0.68-0.90)$ & NR & $0.87(0.75-1.02)$ \\
\hline REWIND ${ }^{54}$ & Dulaglutide & 9,901 & 5.4 years & $0.88(0.79-0.99)$ & $0.93(0.77-1.12)$ & $0.85(0.77-0.93)$ \\
\hline \multicolumn{7}{|l|}{ DPP-4 inhibitor studies } \\
\hline TECOS $^{30-32}$ & Sitagliptin & 14,671 & 3 years & $0.99(0.89-1.11)$ & $1.00(0.83-1.20)$ & NR \\
\hline SAVOR-TIMI $53^{33-36}$ & Saxagliptin & 16,492 & 2 years & $1.00(0.89-1.12)$ & $1.27(1.07-1.51)$ & $1.08(0.96-1.22)$ \\
\hline CARMELINA $^{37}$ & Linagliptin & 6,979 & 2.2 years & $1.02(0.89-1.17)$ & $-0.27(-0.82$ to 0.28$)$ & $1.04(0.89-1.22)$ \\
\hline VERIFY40 & Vildagliptin & 2,001 & 5 years & $0.71(0.42-1.19)$ & NR & NR \\
\hline \multicolumn{7}{|l|}{ SGLT-2 inhibitor studies } \\
\hline EMPA-REG OUTCOME & Empagliflozin & 7,020 & 3.1 years & $0.86(0.74-0.99)$ & $0.65(0.50-0.85)$ & NR \\
\hline CANVAS $^{62}$ & Canagliflozin & 10,142 & 3.6 years (mean) & $0.86(0.75-0.97)$ & $0.67(0.52-0.87)$ & $0.60(0.47-0.77)$ \\
\hline DECLARE-TIMI $58^{83,64}$ & Dapagliflozin & 17,160 & 4.2 years & $0.93(0.84-1.03)$ & $0.73(0.61-0.88)$ & $0.76(0.67-0.87)$ \\
\hline VERTIS ${ }^{65}$ & Ertugliflozin & 8,246 & 3.5 years (mean) & $0.97(0.85-1.11)$ & $0.70(0.54-0.90)$ & $0.81(0.63-1.04)$ \\
\hline SOLOIST-WHF & Sotagliflozin & 1,222 & 9.0 months & NR & $0.64(0.49-0.83)$ & NR \\
\hline SCORED ${ }^{67}$ & Sotagliflozin & 10,584 & 16 months & $0.84(0.72-0.99)$ & $0.67(0.55-0.82)$ & $0.71(0.46-1.08)$ \\
\hline
\end{tabular}

$\mathrm{Cl}=$ confidence interval; DPP-4 = dipeptidyl peptidase-4; GLP-1 = glucagon-like peptide-1; HR = hazard ratio; MACE = major adverse cardiovascular event; NR = not reported; $P O=$ oral administration; $S C=$ subcutaneous administration; SGLT-2 = sodium-glucose cotransporter-2.

and hospitalizations and urgent visits for HF. The median duration of follow-up was 9.0 months. The incidence of the primary endpoint was lower in the sotagliflozin group compared with the placebo group, and this difference was statistically significant (51.0 per 100 patient-years in the sotagliflozin group and 76.3 per 100 patient-years in the placebo group; $\mathrm{HR}, 0.67 ; 95 \% \mathrm{Cl}, 0.52-0.85 ; \mathrm{p}<0.001)$. The incidence of any death or death from CV causes did not differ significantly between the two groups. These findings were consistent between subgroups, including those who enrolled before or after discharge, and those with reduced, mid-range or preserved ejection fraction. A major limitation of the study was that funding from the sponsors was withdrawn before enrolment of the initially planned sample size. However, the trial results suggested that sotagliflozin therapy resulted in fewer incidences of death from CV causes and hospitalization or urgent visits compared with placebo. ${ }^{67}$

The SCORED trial was also conducted to compare sotagliflozin with placebo, but the study population was patients with T2DM and chronic kidney disease and risk for CVD. ${ }^{68}$ A total of 10,584 patients were randomized in the study. The primary endpoint, which was changed during the trial, was eventually a composite of the total number of deaths from CV causes, hospitalizations for HF and urgent visits for HF. The rate of the total primary endpoint was lower in the sotagliflozin group compared with the placebo group (5.6 versus 7.5 events per 100 patient-years, respectively; HR, $0.74 ; 95 \% \mathrm{Cl}, 0.63-0.88 ; \mathrm{p}<0.001$ ). In addition, it is worth mentioning that the original co-primary endpoint of 3-P MACE showed a HR of $0.84(95 \% \mathrm{Cl}, 0.72-0.99)$. The total number of hospitalizations for HF and urgent visits due to HF were also lower in the sotagliflozin group (3.5 versus 5.1 events per 100 patient-years in the sotagliflozin and placebo groups, respectively; HR, 0.67; 95\% $\mathrm{Cl}, 0.55-0.82 ; \mathrm{p}<0.001)$. However, the differences in death from $\mathrm{CV}$ cause and all-cause mortality were not statistically significant between the two groups. Serious adverse events leading to withdrawal from the treatment regimen were not significantly different, but genital mycotic infections, diabetic ketoacidosis and diarrhoea were more common with sotagliflozin treatment. This study had two significant limitations. First, the withdrawal of funding decreased the follow-up duration. Second, the primary endpoint changed during the trial, and this probably affected the statistical power of the study. Overall, the SCORED trial showed that patients with T2DM and chronic kidney disease treated with sotagliflozin were at a lower risk of the composite of deaths from CV causes, hospitalizations for HF and urgent visits for HF than patients receiving placebo. ${ }^{68}$ CVOTs in T2DM are summarized in Table 2. ${ }^{30-7,40,41,43,4,4,47,4,9,50,52-4,45,5,58,62-7}$

\section{Changing the guidelines in diabetes}

New guidelines from the American Diabetes Association (ADA) were published in January 2020 and include recommendations on the use of antidiabetic medication in patients with T2DM and a high CV risk profile. ${ }^{69}$ Several recent CVOTs have published findings regarding CV outcomes of SGLT-2 inhibitors and GLP-1 receptor agonists, although initially these agents were studied as antidiabetic drugs. The results of these CVOTs have shown numerous CV benefits, including reduction in the incidence of major adverse CV events, stroke, MI, CV mortality and hospitalization due to HF. ${ }^{19,70,71}$ Patients with T2DM and established atherosclerotic heart disease or kidney disease are recommended to take SGLT-2 inhibitors or GLP-1 agonists as part of the antidiabetic regimen. Moreover, patients who have an established atherosclerotic disease, yet with multiple CV risk factors or evidence of current diabetic kidney disease, are advised to take SGLT-2 inhibitors as part of protection against a future 3-P MACE incident or future HF 
hospitalizations. Of interest, the ADA recommends that patients with T2DM and established chronic HF (though not specifying whether this refers to patients with reduced ejection fraction or preserved ejection fraction) take SGLT-2 inhibitors to prevent future hospitalizations. ${ }^{69}$

The 2019 guidelines from the ESC, in collaboration with the European Association for the Study of Diabetes (EASD), consider SGLT-2 inhibitors and GLP-1 receptor agonists as first-line treatment for patients with DM and CVD, presenting a different approach compared with the previous 2013 guidelines, which considered metformin to be the gold-standard first-line therapy. 19,72 Interestingly, the 2019 ESC/EASD guidelines state that metformin can be used in obese patients with diabetes, without CVD and at moderate CV risk..$^{19}$ The guidelines also recommend that SGLT-2 inhibitors such as empagliflozin, canagliflozin and dapagliflozin are used for patients with T2DM and CVD, or at very high/high CV risk, in order to reduce $C V$ events. Empagliflozin is also recommended in patients with T2DM and CVD in order to reduce the risk of death. ${ }^{19,73}$ Remarkably, the same recommendations were provided for the GLP-1 receptor agonists, such as liraglutide, semaglutide and dulaglutide for patients with T2DM and CVD, or at very high/high CV risk, in order to reduce CV events. Liraglutide is also recommended in patients with T2DM and CVD, or at very high/high CV risk, in order to reduce the risk of death. ${ }^{19}$ On closer inspection of the updated guidelines, in patients with established CVD and predominant coronary artery disease, GLP-1 receptor agonists with proven $\mathrm{CV}$ benefit may be preferred after metformin, with SGLT-2 inhibitors to be the second choice. Furthermore, for patients with established CVD and predominant HF, SGLT-2 inhibitors should be the first choice after metformin, while GLP-1 agonists with proven $\mathrm{CV}$ benefit should be the second choice. When our primary concern is to reduce hypoglycaemia, DPP-4 inhibitors, GLP-1 receptor agonists, SGLT-2 inhibitors and thiazolidinediones should be preferred. Furthermore, in patients whose body weight is the main concern, GLP-1 receptor agonists or SGLT-2 inhibitors should be chosen. Finally, if cost is a major issue, sulphonylureas or thiazolidinediones may be used after metformin in the treatment of T2DM..$^{73}$ Given that the concept of a sole glucocentric approach to DM management has been modified during the past few years, we need to approach our patients first as a CV entity. Their CV status needs to be identified, and a treatment that has been proven to reduce their $\mathrm{CV}$ risk must be applied, while also targeting tight glucose control in the safest possible way. ${ }^{73}$

Interestingly, in a paper published in 2011, Long and Dagogo-Jack found that approximately $75 \%$ of adults with DM had concomitant hypertension, and that most patients with hypertension had signs of hyperglycaemia. ${ }^{74}$ Several studies over recent years have examined both the microvascular and macrovascular complications related to hypertension and DM, which are interconnected in the vast majority of cases. Many randomized trials in the literature have revealed that optimization of blood pressure has significant cardioprotective advantages, especially in patients with DM. Thus, efforts have focused on the therapeutic management of both diseases, emphasizing pharmacological and lifestyle modifications. ${ }^{74}$

Contrary to the 2013 guidelines, the updated 2019 guidelines do not include a blood pressure target of <140/85 mmHg for all patients; instead, more individualized blood pressure targets are now recommended. ${ }^{72}$ The systolic blood pressure target is now $130 \mathrm{mmHg}$ and $<130 \mathrm{mmHg}$ if well tolerated, but not $<120 \mathrm{mmHg}$. Moreover, in patients with diabetes aged $>65$ years, the systolic blood pressure target should range between
130 and $139 \mathrm{mmHg}$. The suggested target for diastolic blood pressure is $<80 \mathrm{mmHg}$, but not $<70 \mathrm{mmHg} .^{72}$

The 2019 ESC/EASD guidelines reclassified patients with diabetes into moderate, high and very high CVD risk compared with the previous guidelines, which referred to primary and secondary prevention. More specifically, young patients with DM (patients with T1DM, aged $<35$ years or patients with T2DM, aged $<50$ years) with disease duration $<10$ years, without other risk factors, are classified as moderate risk. Patients at high risk for CVD are considered to be those with DM for $\geq 10$ years without target organ damage plus any other additional risk factor. Finally, patients with DM and established CVD, other target organ damage, three or more major risk factors or early-onset T1DM of $>20$ years are stratified as very high risk according to the ESC SCORE charts. $^{74}$ Other revised recommendations include that lifestyle changes should be considered as a primary prevention approach for all patients. ${ }^{19,74}$

Various studies have examined the crucial role of lifestyle interventions in the treatment of prediabetes and the prevention of $\mathrm{CV}$ complications in patients with T2DM. ${ }^{11}$ First, caloric intake reduction is recommended to lower the excess body weight in patients with T2DM. Several publications have shown the benefits of a low caloric intake diet in $\mathrm{HbA} 1$ reduction and improved quality of life. Thus, a Mediterranean diet with olive oil and nuts is suggested. Another lifestyle intervention of high importance is the induction of moderate-to-vigorous physical activity of $\geq 150 \mathrm{~min} /$ week for the management of DM..$^{19}$ As a result, all current publications point out the importance of optimizing and controlling blood glucose levels, lipid profile and blood pressure, especially in patients with T2DM and presence of $\mathrm{CV}$ risk factors. Thus, the prevention of disease progression and improvement of clinical outcomes can be achieved by managing the risk factors responsible for most complications. ${ }^{74}$

\section{Conclusions}

The guidelines for managing the CV risk in patients with DM have been revised. They include many aspects of disease management, including lifestyle modifications, control of glycaemia, lipid profile and blood pressure. ${ }^{75}$ It is well known that T2DM, along with hypertension, obesity and metabolic dysregulations, contribute significantly to the development and progression of both CVD and renal dysfunction. ${ }^{11}$ Regardless of the clinical application of these recommendations, the problem of controlling the progression of CRS in patients with diabetes remains. ${ }^{75}$

Several recent CVOTs have examined the effects of glucose-lowering drugs on the CV and renal systems. Results of these studies revealed that these drugs, as well as their antidiabetic action, have a significant benefit to both $\mathrm{CV}$ and renal outcomes when prescribed to patients with DM. Additionally, another important reason to study novel actions of hypoglycaemic agents has been the unsatisfactory and inadequate regulation of glycaemia, proteinuria and blood pressure levels in the diabetic population..$^{5}$ Thus, the investigation and development of antidiabetic drugs with favourable cardiorenal outcomes are crucial. Interestingly, numerous CVOTs have investigated the role of three new categories of glucose-lowering agents, including SGLT-2 inhibitors, GLP-1 receptor agonists and DPP-4 inhibitors. The results showed that these drugs present diverse cardiorenal effects. However, the pleiotropic effects and the safety of these recent hypoglycaemic drugs should be further explored. ${ }^{15}$ ] 
1. Deepthi B, Sowjanya $K$, Lidiya B, et al. A modern review of diabetes mellitus: an annihilatory metabolic disorder. Journal of In Silico \& In Vitro Pharmacology. 2017:3:1

2. Fan W. Epidemiology in diabetes mellitus and cardiovascular disease Cardiovasc Endocrinol. 2017:6:8-16.

3. Palau V Riera M, Soler MJ. The reno-cardiovascular connection in the patient with diabetes mellitus: what's new? Endocrinol Diabetes Nutr. 2017;64:237-40

4. Koniari K, Nikolaou M, Paraskevaidis I, Parissis J. Therapeutic options for the management of the cardiorenal syndrome. Int J Nephrol. 2010;2011:194910

5. De Rosa S, Arcidiacono B, Chiefari E, et al. Type 2 diabetes mellitus and cardiovascular disease: genetic and epigenetic links. Front Endocrinol (Lausanne). 2018:9:2.

6. Hatamizadeh P, Fonarow G, Budoff M, et al. Cardiorenal syndrome: pathophysiology and potential targets for clinical management. Nat Rev Nephrol. 2013;9:99-111.

7. Rangaswami J, Bhalla V, Blair JEA, et al. Cardiorenal syndrome: classification, pathophysiology, diagnosis, and treatment strategies: a scientific statement from the American Heart Association. Circulation. 2019:139:840-78.

8. Song MK, Davies N, Roufogalis B, Huang THW. Management of cardiorenal metabolic syndrome in diabetes mellitus: a phytotherapeutic perspective. J Diabetes Res. 2014;2014:313718

9. Zelniker TA, Braunwald E. Clinical benefit of cardiorenal effects of sodium-glucose cotransporter 2 inhibitors: JACC state-of-the-art review. J Am Coll Cardiol. 2020;75:435-47.

10. Górriz JL, Soler MJ, Navarro-González JF, et al. GLP-1 receptor agonists and diabetic kidney disease: a call of attention to nephrologists. J Clin Med. 2020;9:947.

11. Jindal A, Garcia-Touza M, Jindal N, et al. Diabetic kidney disease and the cardiorenal syndrome: old disease, new perspectives. Endocrinol Metab Clin North Am. 2013:42:789-808.

12. Kim MK. Treatment of diabetic kidney disease: current and future targets. Korean J Intern Med. 2017;32:622-30.

13. Dhindsa DS, Sandesara PB, Shapiro MD. The intersection of diabetes and cardiovascular disease - a focus on new therapies. Front Cardiovasc Med. 2018;5:160.

14. Nagahisa T, Saisho Y. Cardiorenal protection: potential of SGLT2 inhibitors and GLP-1 receptor agonists in the treatment of type 2 diabetes. Diabetes Ther. 2019;10:1733-52.

15. McHugh KR, DeVore AD, Mentz RJ, et al. The emerging role of novel antihyperglycemic agents in the treatment of heart failure and diabetes: a focus on cardiorenal outcomes. Clin Cardiol. 2018:41:1259-67.

16. Margulies KB, Hernandez AF, Redfield MM, et al. Effects of liraglutide on clinical stability among patients with advanced heart failure and reduced ejection fraction: a randomized clinical trial. JAMA. 2016;316:500-8.

17. Davies MJ, Bain SC, Atkin SL, et al. Efficacy and safety of liraglutide versus placebo as add-on to glucose-lowering therapy in patients with type 2 diabetes and moderate renal impairment (LIRA-RENAL): a randomized clinical trial. Diabetes Care. 2016;39:222-30.

18. Zannad F, Cannon CP, Cushman WC, et al. Heart failure and mortality outcomes in patients with type 2 diabetes taking alogliptin versus placebo in EXAMINE: a multicentre, randomised, double-blind trial. Lancet. 2015;385:2067-76.

19. Cosentino F, Grant PJ, Aboyans V, et al. 2019 ESC Guidelines on diabetes, pre-diabetes, and cardiovascular diseases developed in collaboration with the EASD. Eur Heart J. 2020:41:255-323.

20. Garber AJ. Long-acting glucagon-like peptide 1 receptor agonists: a review of their efficacy and tolerability. Diabetes Care. 2011;34(Suppl. 2):S279-84

21. Górriz JL, Navarro-González JF, Ortiz A, et al. Sodium-glucose cotransporter 2 inhibition: towards an indication to treat diabetic kidney disease. Nephrol Dial Transplant. 2020;35(Suppl. 1):i13-23.

22. UK Prospective Diabetes Study Group. Tight blood pressure control and risk of macrovascular and microvascular complications in type 2 diabetes: UKPDS 38. BM. 1998;317:703-13.

23. Heller SR. A summary of the ADVANCE trial. Diabetes Care 2009;32(Suppl. 2):S357-61.

24. Skyler JS, Bergenstal R, Bonow RO, et al. Intensive glycemic control and the prevention of cardiovascular events: implications of the ACCORD, ADVANCE, and VA Diabetes trials. Diabetes Care. 2009:32:187-92.

25. Duckworth W, Abraira C, Moritz T, et al. Glucose control and vascular complications in veterans with type 2 diabetes. N Engl J Med. 2009;360:129-39.

26. Schnell O, Stand $E$, Catrinoiu D, et al Report from the 4 th Cardiovascular Outcome Trial (CVOT) Summit of the Diabetes \& Cardiovascular Disease (D\&CVD) EASD Study Group. Cardiovasc Diabetol. 2019;18:30

27. Smith RJ, Goldfine AB, Hiatt WR. Evaluating the cardiovascular safety of new medications for type 2 diabetes: time to reassess? Diabetes Care. 2016;39:738-42.

28. McGuire DK, Marx N, Johansen OE, et al. FDA guidance on antihyperglycaemic therapies for type 2 diabetes: one decade later. Diabetes Obes Metab. 2019:21:1073-8.

29. Cefalu WT, Kaul S, Gerstein HC, et al. Cardiovascular outcomes trials in type 2 diabetes: where do we go from here? Reflections from a diabetes care editors' expert forum Diabetes Care. 2018;41:14-31.
30. Nauck MA, MCGuire DK, Pieper KS, et al. Sitagliptin does not reduce the risk of cardiovascular death or hospitalization for heart failure following myocardial infarction in patients with diabetes: observations from TECOS. Cardiovasc Diabetol. 2019:18:116

31. Davis TME, Mulder H, Lokhnygina Y, et al. Effect of race on the glycaemic response to sitagliptin: insights from the Trial Evaluating Cardiovascular Outcomes with Sitagliptin (TECOS). Diabetes Obes Metab. 2018;20:1427-34.

32. Green JB, Bethel MA, Armstrong PW, et al. Effect of sitagliptin on cardiovascular outcomes in type 2 diabetes. N Engl I Med. 2015;373:232-42

33. Kumbhani DJ. Saxagliptin assessment of vascular outcomes recorded in patients with diabetes mellitus - thrombolysis in myocardial infarction 53 - SAVOR-TIMI 53. 2017. Available at: www.acc.org/latest-in-cardiology/clinicatrials/2014/05/20/23/04/savor-timi-53 (accessed 17 November 2021).

34. European Society of Cardiology. SAVOR-TIMI 53 sets new standard for cardiovascular outcome trials in diabetes. Saxagliptin demonstrated better control of glucose compared with placebo 2013. [Press releasel. Available at: wnw escardio. org/The-ESC/Press-Office/Press-releases/SAVOR-TIMI-53-SetsNew-Standard-for-Cardiovascular-Outcome-Trials-in-Diabetes (accessed 17 November 2021).

35. Udell JA, Bhatt DL, Braunwald E, et al. Saxagliptin and cardiovascular outcomes in patients with type 2 diabetes and moderate or severe renal impairment: observations from the SAVOR-TIMI 53 Trial. Diabetes Care. 2015;38:696-705.

36. Singh AK, Singh R. SAVOR-TIMI to DECLARE-TIMI: a review on cardiovascular outcome trials of incretin-modulators and gliflozins. Indian J Endocrinol Metab. 2019;23:175-83.

37. Mosenzon O, Leibowitz G, Bhatt DL, et al. Effect of saxagliptin on renal outcomes in the SAVOR-TIMI 53 trial. Diabetes Care 2017;40:69-76.

38. Rosenstock $\rfloor$ Perkovic $V$, Johansen $O E$, et al. Effect of linagliptin vs placebo on major cardiovascular events in adults with type 2 diabetes and high cardiovascular and renal risk: the CARMELINA randomized clinical trial. JAMA. 2019;321:69-79.

39. ClinicalTrials.gov. Cardiovascular and Renal Microvascular
Outcome Study With Linagliptin in Patients With Type 2 Diabetes Mellitus (CARMELINA). ClinicalTrials.gov Identifier: NCT01897532. Available at: https://clinicaltrials.gov/ct2/show NCT01897532 (accessed 17 November 2021).

40. Endocrine Today. CARMELINA, CAROLINA shed new light on CV safety for linagliptin, glimepiride. 2019. Available at: www.healio.com/endocrinology/diabetes/news/prin endocrine-today/\%7Ba3c4a49c-4dd6-466d-bc72 $30 \mathrm{e} 26 \mathrm{c} 88914 \mathrm{~b} \% 7 \mathrm{D} / \mathrm{carmelina-carolina-shed-new-light-on-cv-}$ safety-for-linagliptin-glimepiride? page $=3$ (accessed 17 November 2021)

41. Matthews DR, Paldánius PM, Proot $P$, et al. Glycaemic durability of an early combination therapy with vildagliptin and metformin versus sequential metformin monotherapy in newly diagnosed type 2 diabetes (VERIFY): a 5-year, multicentre, randomised, double-blind trial. Lancet. 2019;394:1519-29.

42. Canadian Agency for Drugs and Technologies in Health. Clinical review report: Lixisenatide (Adlyxine): (Sanofi-Aventis Canada Inc.): Indication: As an adjunct to diet and exercise to improve glycemic control in adult patients with type 2 diabetes mellitus in combination with metformin, a sulfonylurea (alone or with metformin), pioglitazone (alone or with metformin), a basal insulin (alone or with metformin), when the therapy listed above does not provide adequate glycemic control. 2017. Available at: www.ncbi.nlm.nih.gov/books/NBK535139/ (accessed 11 October 2021).

43. ClinicalTrials. gov. Evaluation of Cardiovascular Outcomes in Patients With Type 2 Diabetes After Acute Coronary Syndrome During Treatment With AVE0010 (Lixisenatide) (ELIXA). ClinicalTrials.gov Identifier: NCT01147250. Available at: https://clinicaltrials.gov/ct2/show/r
(accessed 17 November 2021).

44. Muskiet MHA, Tonneijck L. Huang Y, et al. Lixisenatide and rena outcomes in patients with type 2 diabetes and acute coronary syndrome: an exploratory analysis of the ELIXA randomised, placebo-controlled trial. Lancet Diabetes Endocrinol. 2018;6:859-69.

45. Zinman B, Nauck MA, Bosch-Traberg H, et al. Liraglutide and glycaemic outcomes in the LEADER trial. Diabetes Ther. 2018;9:2383-92

46. Marso SP, Poulter NR, Nissen SE, et al. Design of the liraglutide effect and action in diabetes: evaluation of cardiovascular outcome results (LEADER) trial. Am Heart J. 2013;166:823-30.

47. Marso SP, Daniels GH, Frandsen KB, et al. Liraglutide and cardiovascular outcomes in type 2 diabetes. N Eng/ J Med. 2016;375:311-22

48. Holman RR, Bethel MA, Mentz RJ, et al. Effects of once-weekly exenatide on cardiovascular outcomes in type 2 diabetes. N Engl J Med. 2017;377:1228-39.

49. Torekov SS. Glucagon-like peptide-1 receptor agonists and cardiovascular disease: from LEADER to EXSCEL. CardiovasC Res. 2018;114:70-1.

50. Marso SP, Bain SC, Consoli A, et al. Semaglutide and cardiovascular outcomes in patient

51. Cordiner R, Fisher M, Drummond RS. SUSTAIN-6: cardiovascular safety of a once-weekly GLP-1 receptor agonist. Pract Diabetes. 2016;33:266-8.

52. Vergès $B$, Charbonnel $B$. After the LEADER trial and SUSTAIN- 6 , how do we explain the cardiovascular benefits of some GLP-1 receptor agonists? Diabetes Metab. 2017:43(Suppl. 1):2S3-2S12.

53. Husain M, Birkenfeld AL, Donsmark M, et al. Oral semaglutide and cardiovascular outcomes in patients with type 2 diabetes. N Engl J Med. 2019;381:841-5

54. Hernandez AF, Green JB, Janmohamed S, et al. Albiglutide and cardiovascular outcomes in patients with type 2 diabetes an cardiovascular disease (Harmony Outcomes): a double-blind, randomised placebo-controlled trial. Lancet. 2018;392:1519-29.

55. Gerstein HC, Colhoun HM, Dagenais GR, et al. Dulaglutide and cardiovascular outcomes in type 2 diabetes (REWIND): a double-blind, randomised placebo-controlled trial. Lancet. 2019:394:121-30

56. Rastogi A, Bhansali A. SGLT2 inhibitors through the windows of EMPA-REG and CANVAS trials: a review. Diabetes Ther. 2017:8:1245-51.

57. Fitchett D, Inzucchi SE, Cannon CP, et al. Empagliflozin reduced mortality and hospitalization for heart failure across the spectrum of cardiovascular risk in the EMPA-REG OUTCOME trial. Circulation. 2019;139:1384-95

58. Wanner C. Summary. What are the key lessons from the EMPA-REG OUTCOME trial? 2018. Available at: https://pacecme.org/2018/07/30/summary-what-are-the-key-lessons-fromthe-empa-reg-outcome-trial/ (accessed 17 November 2021).

59. Zinman B, Wanner C, Lanchin J, et al. Empagliflozin, cardiovascular outcomes, and mortality in type 2 diabetes N Eng/ J Med. 2015;373:2117-28.

60. Carbone S, Dixon DL. The CANVAS Program: implications of canagliflozin on reducing cardiovascular risk in patients with type 2 diabetes mellitus. Cardiovasc Diabetol. 2019;18:64.

61. Januzzi I Jr Bavry AA Canagliflozin Cardiovascular Assessment Study - CANVAS. 2019. Available at: www.acc.org/ latest-in-cardiology/clinical-trials/2017/06/12/16/25/canvas (accessed 17 November 2021).

62. Shah SR, Najim NI, Abbasi Z, et al. Canagliflozin and cardiovascular disease - results of the CANVAS trial. J Community Hosp Intern Med Perspect. 2018;8:267-8.

63. Mahaffey KW, Neal B, Perkovic V, et al. Canagliflozin for primary and secondary prevention of cardiovascular events: results from the CANVAS Program (Canagliflozin Cardiovascular Assessment Study). Circulation. 2018;137:323-34.

64. Wiviott SD, Raz I, Bonaca MP, et al. Dapagliflozin and cardiovascular outcomes in type 2 diabetes. N Eng/ I Med. 2018;380:347-57

65. Al-Bazz DY, Wilding JP. Dapagliflozin and cardiovascular outcomes in patients with type 2 diabetes. Future Cardiol. 2020;16:77-88.

66. Cannon CP, Pratley R, Dagogo-Jack $\mathrm{S}$, et al. Cardiovascular outcomes with ertugliflozin in type 2 diabetes. N Eng/ I Med. 2020;383:1425-35

67. Bhatt DL, Szarek M, Steg PG, et al. Sotagliflozin in patients with diabetes and recent worsening heart failure. N Eng/ I Med. 2021;384:117-28.

68. Bhatt DL, Szarek M, Pitt B, et al. Sotagliflozin in patients with diabetes and chronic kidney disease. N Engl I Med. 2021;384:129-39

69. American Diabetes Association. Cardiovascular disease and risk management: standards of medical care in diabetes-2020. Diabetes Care. 2020;43(Suppl. 1):S111-34

70. GlobalData Healthcare. ESC updates first-line treatment guidelines for type 2 diabetics with cardiovascular disease 2019. Available at: www.pharmaceutical-technology.com/ comment/esc-updates-first-line-treatment-guidelines-for-type2-diabetics-with-cardiovascular-disease/ (accessed 17 November 2021)

71. European Society of Cardiology. 2019 guidelines on diabetes, pre-diabetes and cardiovascular diabetes developed in collaboration with the EASD. 2019. Available at: www.escardio.org/Guidelines/Clinical-Practice-Guidelines/ Diabetes-Pre-Diabetes-and-Cardiovascular-Diseasesdeveloped-with-the-EASD (accessed 17 November 2021)

72. Rydén L, Grant PJ, Anker SD, et al. ESC Guidelines on diabetes, pre-diabetes, and cardiovascular diseases developed in collaboration with the EASD: the task force on diabetes, pre-diabetes, and cardiovascular diseases of the European Society of Cardiology (ESC) and developed in collaboration with the European Association for the Study of Diabetes (EASD). Eur Heart J. 2013;34:3035-87.

73. Physicians' Academy for Cardiovascular Education. New ESC/EASD guidelines on diabetes no longer regard metformin as first-line therapy. Available at: https://pace-cme. org/2019/09/03/new-esc-easd-guidelines-on-diabetes-nolonger-regard-metformin-as-first-line-therapy/ (accessed 17 November 2021)

74. Long AN, Dagogo-Jack S. Comorbidities of diabetes and hypertension: mechanisms and approach to target organ protection. J Clin Hypertens (Greenwich). 2011;13:244-51.

75. Martinez-Castelao A, Soler MJ, Navarro-González JF, Górriz JL. Will the new molecules be effective in renal and cardiovascular protection in diabetes mellitus and diabetic kidney disease? Nefrologia (Engl Ed). 2019;39:3-10. 